\title{
Harmonic Elimination of a Photo-Voltaic Based Cascaded H-Bridge Multilevel Inverter Using ANFIS for Non-Linear Loads
}

\author{
Rajarajeshwari.C ${ }^{1}$, Sivasundari. $K^{2}$, Shalini.S ${ }^{3}$, Karuppa Samy.A ${ }^{4}$ \\ UG Student, Department of Electrical and Electronics Engineering, Kamaraj College of Engineering and Technology, \\ Virudhunagar, Tamil Nadu, India ${ }^{1,2,3}$ \\ Assistant Professor, Department of Electrical and Electronics Engineering, Kamaraj College of Engineering and \\ Technology, Virudhunagar, Tamil Nadu, India ${ }^{4}$
}

\begin{abstract}
In this study, a multilevel inverter was designed and implemented to operate a stand-alone solar photovoltaic system. The proposed system uses pulse-width modulation (PWM) in the multilevel inverter to convert DC voltage from battery storage to supply AC loads. In the PWM method, the effectiveness of eliminating low-order harmonics in the inverter output voltage is studied and compared to that of the sinusoidal PWM method. This work also uses adaptive neuro fuzzy inference (ANFIS) to predict the optimum modulation index and switch angles required for a five level cascaded H-bridge inverter with improved inverter output voltage. The data set for the ANFIS-based analysis was obtained with the Newton-Raphson (NR) method. The proposed predictive method is more convincing than other techniques in providing all possible solutions with any random initial guess and for any number of levels of a multilevel inverter. The simulation results prove that the lower-order harmonics are eliminated using the optimum modulation index and switching angles. An experimental system was implemented to demonstrate the effectiveness of the proposed system.
\end{abstract}

Keywords: Adaptive neuro-fuzzy inference system, Harmonic elimination, Multi-level inverters, Newton -Raphson method.

\section{INTRODUCTION}

The flexibility of interfacing renewable energy sources such as photo-voltaic arrays and wind turbines has made cascade multilevel inverter more reliable in drives and smart grid applications. But the main challenge in such applications is the elimination of harmonics produced due to switching of large number of power electronics switches present in the cascaded multilevel inverter topology. Multilevel inverters play a major role in numerous applications such as medium-voltage drives, renewable energy system, grid interfaces, and flexible AC transmission devices. A multilevel inverter is a familiar power electronic converter that can synthesize a desired output voltage from several levels of DC voltages as input source.

Much research work has been devoted to equal DC sources and little to unequal DC sources in the multilevel inverter circuit topologies. Based on these identical voltage levels and proper pulse-width modulation (PWM) control of the switching angles, a staircase waveform can be synthesized. The reduction of switching losses in the inverter devices due to the on and off switch operation during one fundamental cycle is one of the major benefits of the staircase output waveform. Moreover, with reduced switching frequencies, low-order frequency harmonics can be observed in the staircase output voltage.
Although many approaches have focused on eliminating low-order frequency harmonics, the selective harmonic elimination (SHE) PWM method is preferred widely in multilevel inverters. The selective harmonic elimination pulse-width modulation switching (SHEPWM) technique can be used to compute switching angles by solving the nonlinear transcendental equations characterizing harmonics. Many iterative techniques have been reported to solve the nonlinear transcendental equations resulting in only one solution set or multiple solutions by assuming a proper initial guess. Several traditional methods can be used to estimate switching angles; however, a few of them may be computationally expensive. As soft computing techniques such as artificial neural networks (ANNs) and adaptive neuro-fuzzy inference system (ANFIS) learn and adapt to a great diversity of data, it can be an excellent option to estimate switching angles.

\section{OBJECTIVES}

The major concern in the harmonic elimination problem in the non-equal dc sources system is the solution of the nonlinear transcendental equations. However, the proposed method can solve this problem in a simple manner, by just converting non-equal dc sources into equal 
Vol. 6, Issue 3, March 2017

dc source using the ANFIS (Adaptive Neuro Fuzzy Inference System)/Constant Voltage Maximum Power Point Tracker algorithm for the Photo-voltaic system.

\section{MOTIVATIONS}

Dramatically decrease the THD (Total Harmonic Distortion) of the output voltage of a cascade H-bridge multilevel inverters with induction motor drive. Further, it was concluded that the ANFIS-SHE algorithm gives better performance in THD minimization according to the IEEE519 standard ( $5 \%$ ) compared to the conventional NRSHE algorithm, as PSOSHE algorithm recorded a line voltage THD of $3.7 \%$ for a5 level cascaded

$\mathrm{H}$-bridge inverter for a modulation index of 0.5 . Thus, this system is well suited for solar based induction motor drive applications and in smart grids where renewable sources of energy such as solar, wind and fuel cells need to be interfaced with grid. Further, this research is prospective to future research in the area of high quality energy transfer using power converters.

\section{PROBLEM DEFINITION}

This work mainly aims at developing an algorithm for minimizing the THD of the cascaded multilevel inverter powered by a PV array. The output voltage THD should be $5 \%$ and the magnitude of the individual harmonics should be $-3 \%$. The major concern in the harmonic elimination problem in the non-equal dc sources system is the solution of the nonlinear transcendental equations. However, the proposed method can solve this problem in a simpler manner, by just converting non-equal dc sources into equal dc source using the ANFIS (Adaptive Neuro Fuzzy Inference System)/Constant Voltage Maximum Power Point Tracker algorithm for the Photo-voltaic system. These optimal firing angles are found offline and then used online using look up table and PLL (phase lock loop) control for firing the solar powered cascaded multilevel inverter. The block diagram of the proposed harmonic elimination system of a cascade multilevel inverter powered by a PV array.

\section{BLOCK DIAGRAM}

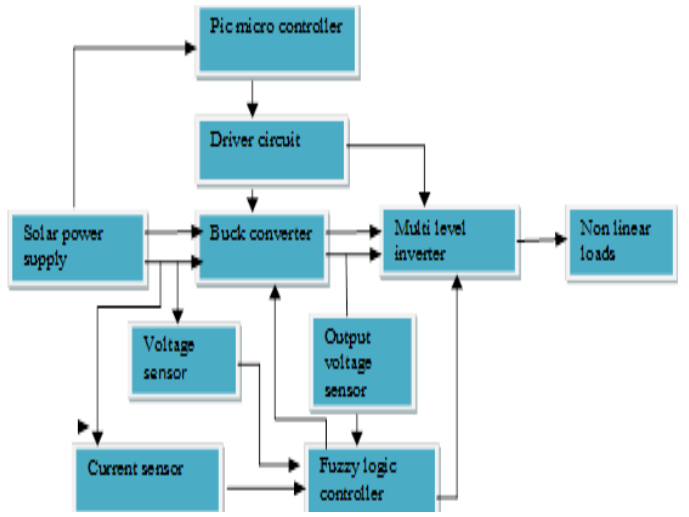

Fig 1. Block diagram
Fig 1 shows the block diagram of Harmonic elimination of a photo-voltaic based cascaded H-bridge multilevel inverter using ANFIS for non-linear loads.

\section{SIMULATION DIAGRAM}

Fig 2 shows the simulation diagram of Harmonic elimination of a photo-voltaic based cascaded H-bridge multilevel inverter using ANFIS for non-linear loads.

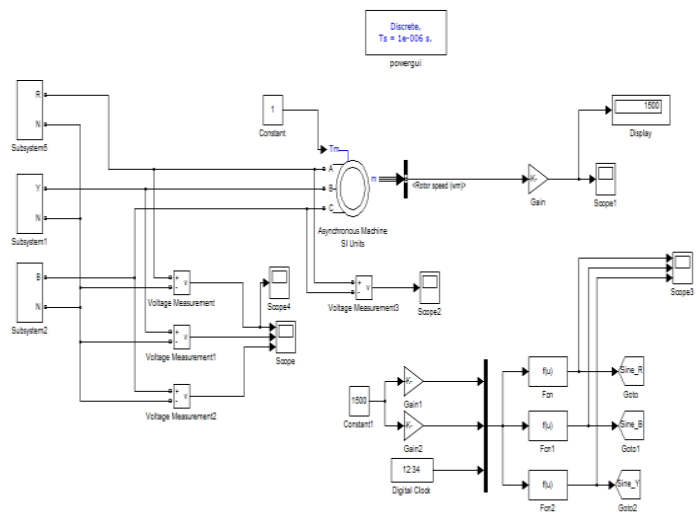

Fig 2. Simulation diagram

Simulation Result

Fig 3 shows the simulation result of an Harmonic elimination of a photo-voltaic based cascaded $\mathrm{H}$-bridge multilevel inverter using ANFIS for non-linear loads.

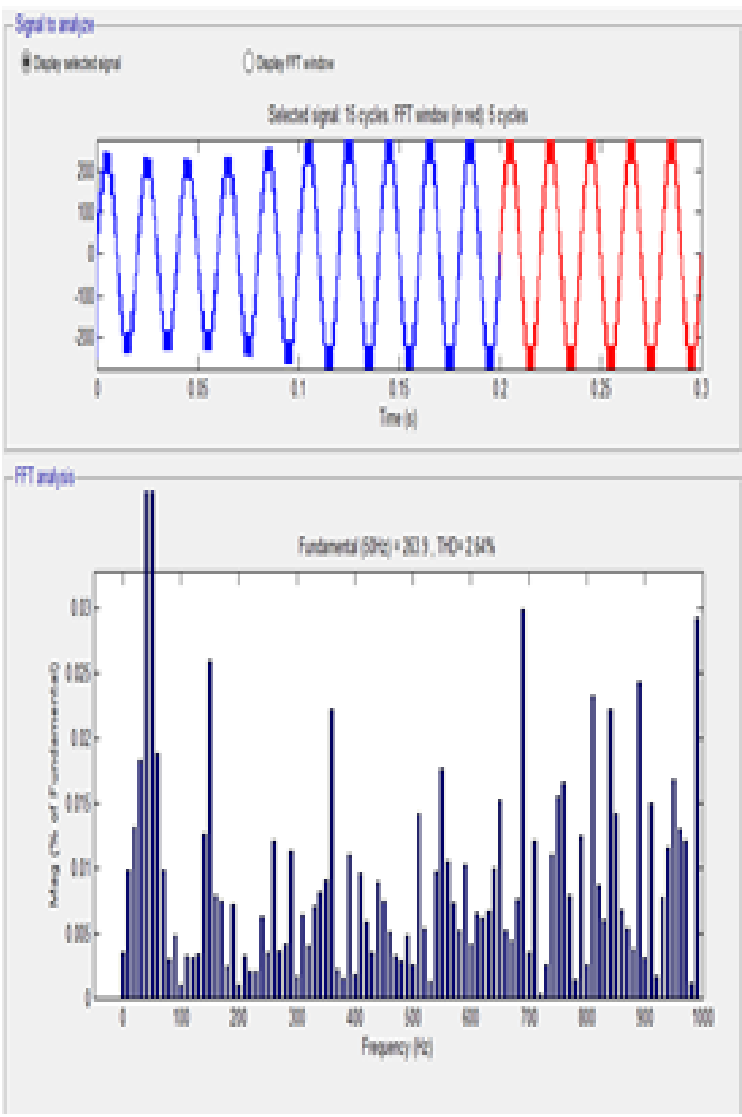

Fig 3. Simulation result 


\section{PRPOSED TECHNIQUES}

A. Adaptive Neuro Fuzzy Inference System (Anfis)-Fuzzy Controller

The performance of Fuzzy logic controller is well documented for improvements of both transient and steady state performances. The function of fuzzy logic controller is very useful since exact mathematical model of it isnot required. The schematic diagram of fuzzy logic control system is shown in Fig 4.

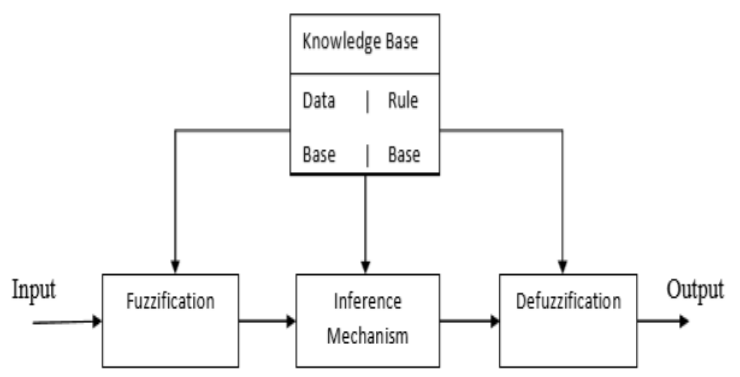

Fig 4. Fuzzy logic controller

It can be divided into four main functional blocks namely Knowledge base, Fuzzification, Inference mechanism and Defuzzification. The knowledge base is composed of database and rule-base. The data-base, consisting of input and output membership functions, provides information for appropriate fuzzification operations, the inference mechanism and defuzzification. The rule-base consists of a set of linguistic rules relating the fuzzy input variables to the desired control actions. Fuzzification converts a crisp input signal, into fuzzified signals that can be identified by level of Input, Output Knowledge. The inference mechanism uses the collection of linguistic rules to convert the input conditions to fuzzified output. Finally, the defuzzification converts the fuzzy outputs to crisp output signals (Kerroucheet al 2009).

\section{B. Fuzzification}

Fuzzification converts a crisp input signal, into fuzzified signals that can be identified by level of membership in the fuzzy sets. Fuzzy logic uses linguistic variables instead of numerical variables. In a control system, error between reference and output can be labelled as very small (VS), Small(S), Medium (M), Large (L) and VeryLarge (VL). The process of converting a numerical variable (real number) convert to a linguistic variable (fuzzy number) is called fuzzification.

\section{C.Inference Mechanism}

It works on the concept of fuzzy if-then rules and fuzzy reasoning. It has a fruitful outcome in many fields such as automatic control, data classification, decision analysis, expert systems, robotics etc. It implements a nonlinear mapping from its input space to output space. The control rules are evaluated by inference mechanism. There are mainly two forms of inference mechanism available (Nagewara Raoetal 2010).
- Mamdani Fuzzy Inference System

- Sugeno Fuzzy Inference System

The basic difference between the two methods is in obtaining the consequent of fuzzy rules. Mamdani FIS uses fuzzy sets for the rule consequents and Sugeno FIS uses linear functions of input variables. Mamdani FIS is universally accepted. Decomposition and aggregation operations are done in the inference system. If the rule is compound in nature, it is converted into a simple canonical rule form. There should be more than one rule available in the rule base system. In aggregation, the overall consequents from the individual consequents are obtained.

\section{D.Knowledge Base}

The knowledge base is composed of data-base and rulebase. The data-base, consisting of input and output membership functions, provides information for appropriate fuzzification operations. Rule base involves defining the rules represented as a set of if then rules, for example if (alpha0 is very small 'VS') and (alpha1 is very small 'VS') and (alpha2 is very small 'VS') then (V1 is very large 'VL') (V3 is large ' $L$ ') (V5 is medium 'M').

\section{E.Data Base}

The Database stores the definition of the membership function required by fuzzier and defuzzifier. The membership to accommodate various "degrees of membership" on the real continuous interval is $[-1,1]$. But there are infinite number of values in between the end points $[-1,1]$, which can represent various degrees of membership for an element. A crisp set has a unique membership function, whereas a fuzzy set can have an infinite number of membership functions to represent it. The main power and strength of membership function is that it employs some amount of overlap. The various form of membership function are

- S membership function

- L membership function

- Triangular membership function

- Gaussian membership function

- Trapezoidal membership function

\section{F. Rule Base}

In conventional controllers, we have control gains or control laws which are combination of numerical values. In FLC, the equivalent term is rules and they are linguistic in nature. IF premise (antecedent- input)... ...THEN conclusion (consequent- output). This form of knowledge representation, characterized as shallow knowledge, is quite appropriate in the context of linguistics because it expresses human empirical and heuristic knowledge in one's own language of communication. The IF-THEN rules can be made as compound rules by adding AND in between IF-THEN. Then, the fuzzy rules become as compound rules like IF.....AND......AND.....THEN. There are mainly four approaches in the formation of fuzzy rules from the experience and control engineering 
knowledge of the experts. From the behaviour of the $h$. In the aggregation of fuzzy outputs 'and' operation is human operator working in the process. Using a fuzzy performed on the rule outputs.

model of a process. Obtaining a relationship through i. Centroid method of defuzzification is used in this work. experience or simulation with a learning process.

A typical rule can be written as follows; If e is $\mathrm{A}$ and ce is $\mathrm{B}$ then output is $\mathrm{C}$ Where $\mathrm{A}, \mathrm{B}, \mathrm{C}$ are the labels of linguistic variables of error (e), Change of error (ce) and output respectively. Here e, ce and output represents degree of membership.

\section{G.Defuzzfication}

The output generated by fuzzy controller must be crisp which is used to control the drive unit and thus accomplished by the defuzzification block. The rules of FLC generate required output in a linguistic variable (Fuzzy Number), according to real world requirements, linguistic variables have to be transformed to crisp output (Real number). The choices available for defuzzification are numerous. So far the choice of strategy is a compromise between accuracy and computational intensity.

Many defuzzification strategies are available, such as, the weight average criterion, the mean-max membership, and centre-of-area (centroid) method. The defuzzification technique used here is based upon centroid method.

\section{FUZZY LOGIC CONTROLLER FOR SWITCHING ANGLE SELECTION}

Fuzzy logic controller is designed for the optimum selection of switching angles for harmonic mitigation in multilevel inverter. The following steps were involved in the design procedure.

a. The input variables are the switching angles and the output variables are the harmonic voltage.

b. The universe of discourse for the input and output variables are portioned into five intervals. The linguistic variables are Very Small(VS), Small(S), Medium(M), Large(L), Very Large(VL). The range for the input is $[0,90]$ and is defined as VS $(0,18), \mathrm{S}(18,36), \mathrm{M}(36,54)$, $\mathrm{L}(54,72)$, $\mathrm{VL}(72,90)$; range for the control output is [$300,300]$ and is defined as $\operatorname{VS}(-300,-180), \mathrm{S}(-180,-60)$, M(-60,60), L(60,180), VL(180,300).

c. Triangular membership function is taken for the input and output variables and they are designed as shown in Fig 5.

d. The Rule base stores the linguistic control rules required by rule evaluator (decision making logic).In the proposed work three inputs (alpha0, alpha1 and alpha2) were used with each input having five membership functions (Very Small (VS), Small(S), Medium (M), Large (L), VeryLarge (VL)).So totally 125 rules were formed (Number of inputs*square of number of membership functions).

e. No normalization is done for the input and output variables.

f. The inputs are applied.

g. Fuzzy approximate reasoning is applied to the above inputs.

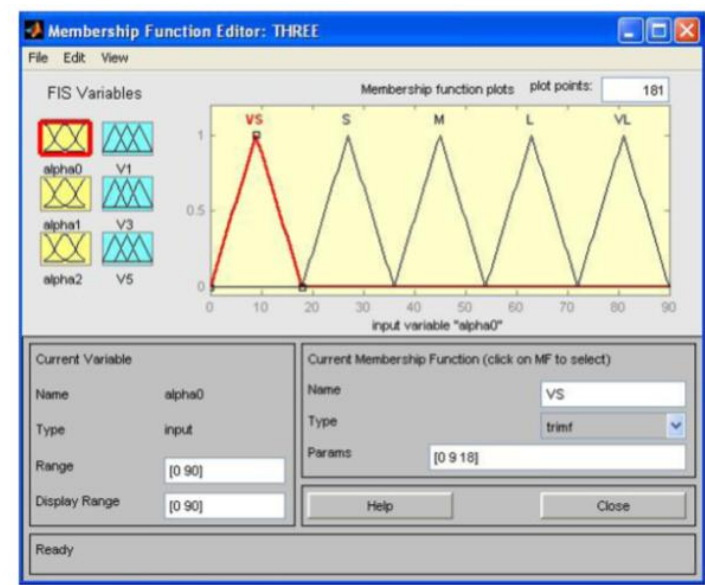

Fig 5. Membership function

\section{MULTI-LEVEL INVERTER}

The multi-level inverter has been introduced since 1975 as alternative in high power and medium voltage situations. The Multi-level inverter is like an inverter and it is used for industrial applications as alternative in high power and medium voltage situations.The need of multilevel converter is to give a high output power from medium voltage source. Sources like batteries, super capacitors, solar panel are medium voltage sources. The multi-level inverter consists of several switches. Where the arrangements of switching angle is very important.

A. Types of Multilevel Inverter

- Diode clamped multilevel inverter

- Flying capacitors multilevel inverter

- Cascaded H- bridge multilevel inverter

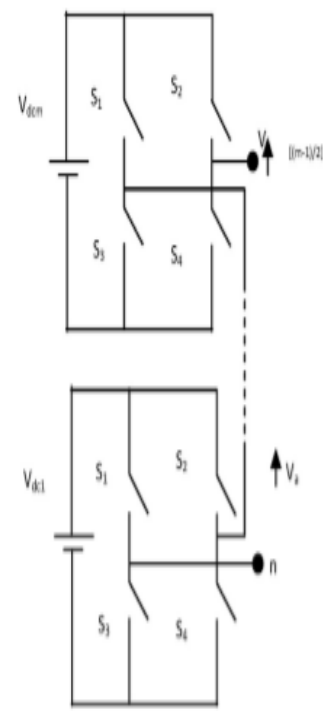

(a)

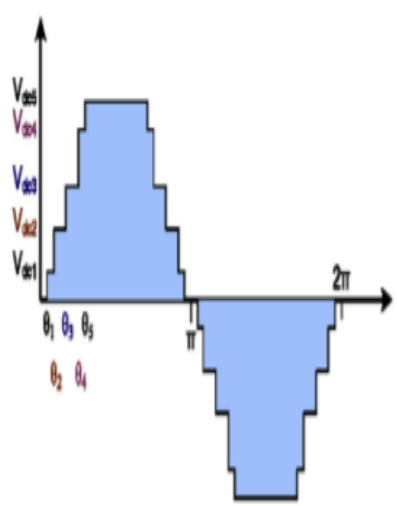

Fig 6. H-bridge inverter Multi-level inverter 
Here we use cascaded H-bridge multilevel inverter because it needs less number of components compared with diode clamped and flying capacitor inverters. The price and weight of the inverterare less than those of the two inverters. Soft-switching is possible by the some of the new switching methods. Multilevel cascade inverters are used to eliminate the bulky transformer required in case of conventional multi-phase inverters, clamping diodes required in case of diode clamped inverters and flying capacitors required in case of flying capacitor inverters. But these require large number of isolated voltages to supply the each cell.

B. Applications of Cascaded H-Bridge Multilevel Inverter

- Motor drives

- Active filters

- Electric vehicle drives

- DC power source utilization

- Power factor compensators

- Back to back frequency link systems

- Interfacing with renewable energy resources.

C. Advantages of Multilevel Inverter

- Common Mode Voltage

The multilevel inverters produce common mode voltage, reducing the stress of the motor and don't damage the motor.

\section{- Input Current}

Multilevel inverters can draw input current with low distortion.

- Switching Frequency

The multilevel inverter can operate at both fundamental switching frequencies that are higher switching frequency and lower switching frequency. It should be noted that the lower switching frequency means lower switching loss and higher efficiency is achieved.

- Reduced harmonic distortion

Selective harmonic elimination technique along with the multi-level topology results the total harmonic distortion becomes low in the output waveform without using any filter circuit

\section{HARDWARE}

Fig 7 shows the hardware setup of Harmonic elimination of a photo-voltaic based cascaded $\mathrm{H}$-bridge multilevel inverter using ANFIS for non-linear loads

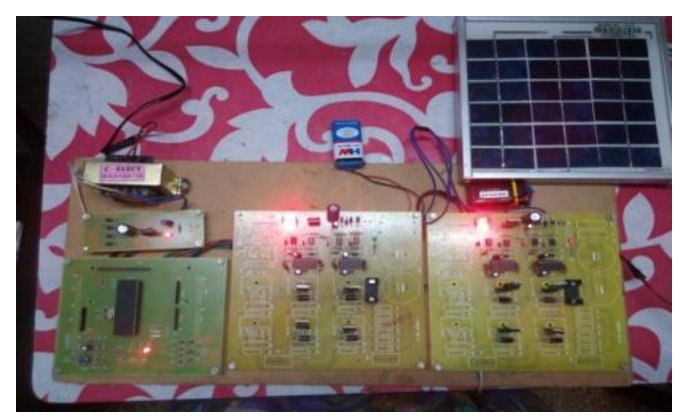

Fig 7. Hardware setup

\section{CONCLUSION}

The total harmonic distortions for both the cases were recorded. The simulation and experimental results depicted that the proposed NR and ANFIS based selective harmonic elimination algorithms can eliminate the lower order harmonics. And thereafter, dramatically decrease the THD (total harmonic distortion) of the output voltage of a cascade H-bridge multilevel inverters with induction motor drive. Further, it was concluded that the ANFISSHE algorithm gives better performance in THD minimization according to the IEEE-519 standard (5\%) compared to the conventional NR-SHE algorithm, as ANFIS-SHE algorithm recorded a line voltage THD of $3.7 \%$ for an 11 level cascaded H-bridge inverter for a modulation index of 0.5 . Thus, this system is well suited for solar based non-linear load applications and in smart grids where renewable sources of energy such as solar, wind and fuel cells need to be interfaced with grid. Further, this research is prospective to future research in the area of high quality energy transfer using power converters.

\section{REFERENCES}

[1] Debnath Suman, Ray Rup Narayan. THD optimization in 13 level photovoltaic inverter using genetic algorithm. Int J Eng

[2] MaruthuPandiPerumal, DevarajanNanjudapan. Performance enhancement of embedded system based multilevel inverter using genetic algorithm.

[3] Venkatramanan CB, Jayakumar KS, Yuvarani B. SHE-PWM control for cascaded voltage source multilevel inverter based on GA optimization.

[4] MaruthuPandi P, Devarajan N. Optimization of power quality in cascaded multilevel inverter-genetic algorithm approach. In: Conf. Rec. IEEE 2nd Int. Conf. on Computing, Communication and Networking Technologies.

[5] OzpineciBurak, Tolbert Leon M, Chiasson John N. Harmonic optimization of multilevel converters using genetic algorithms.

[6] Al Othman AK, Abdelhamid TH. Elimination of harmonics in multilevel inverters with non-equal dc sources using PSO. Elsevier J Energy Convers Manag

[7] Taghizadeh H, TarafdarHagh M. Harmonic elimination of cascade multilevel inverters with non-equal dc sources using particle swarm optimization. 